\title{
ON THE FIELD OF A 2-BLOCK. II
}

\author{
B. G. BASMAJI
}

\begin{abstract}
Every real 2-block $B$ of a finite metabelian group contains an irreducible character $\theta$ such that $Q(B)=Q(\theta)$.
\end{abstract}

Let $B$ be a $p$-block of a finite metabelian group $G$ and let $\zeta$ be a primitive $|G|$ th root of unity over the rationals $Q$. If $\tau \in \mathcal{G}(Q(\zeta) / Q)$, the Galois group, it was shown in [3] that the set of all $\chi^{\tau}$, where $\chi$ is an irreducible ordinary character of $B$, form all the irreducible ordinary characters of some block denoted by $B^{\tau}$. Let $\mathcal{H}(B)=\{\tau \in$ $\left.\mathcal{G}(Q(\zeta) / Q) \mid B^{\tau}=B\right\}$ and $Q(B)$ the subfield of $Q(\zeta)$ fixed by $\mathcal{H}(B)$. Assuming $B$ is a 2-block, it was proved in [3] that $Q(B)=Q(\theta)$ for some irreducible ordinary character $\theta \in B$ if the 2-Sylow subgroup of $G^{\prime}$ is cyclic $\left(G^{\prime}\right.$ abelian). Such an equality does not hold in general. However, in this paper we prove that if $B$ is real then $Q(B)=Q(\theta)$ for some irreducible $\theta \in B$. In particular, this gives another aspect of real 2-blocks studied in Gow [4], where it was proved that every real 2-block of any finite group has an irreducible real character.

THEOREM. Let $B$ be a real 2-block of a finite metabelian group $G$. Then $B$ contains an irreducible 2-rational character $\theta$ of height zero such that $Q(B)=Q(\theta)$.

Proof. We use the results in [1 and 2]. Let $P$ be the 2-Sylow subgroup of the commutator group $G^{\prime}, G^{\prime}=P \times G_{1}$, and $H=P \times \Lambda, \Lambda \subseteq G_{1}$, such that $G^{\prime} / H$ is cyclic. For any subgroup $L$ of $G^{\prime}$ let $K(L) \supseteq G^{\prime}$ be a subgroup of $N(L)$, the normalizer of $L$ in $G$, such that $K(L) / L$ is a maximal abelian subgroup of $N(L) / L$. Fix $K(\Lambda)$ and if $\Lambda \subseteq L \subseteq H$, pick $K(H) \supseteq K(L) \supseteq K(\Lambda)$. Let $\sigma$ be a linear 2-modular representation of $K(\Lambda), S=\operatorname{ker} \sigma$, and $S \cap G^{\prime}=H$. Let $B(\sigma, H)$ be the collection of all representations $T^{\prime G}$ where $T^{\prime}$ is a linear (ordinary) representation of $K(L)$, ker $T^{\prime} \cap G^{\prime}=L$, with the modular representation $\bar{T}_{K(\Lambda)}^{\prime}$ being $G$-conjugate to $\sigma$ (notations are the same as in [2]), and $L$ runs over all subgroups of $G^{\prime}, G^{\prime} / L$ cyclic, $\Lambda \subseteq L \subseteq H$. Include in $B(\sigma, H)$ the composition factors (and their Brauer characters) of the modular representations $\bar{T}^{\prime} G$, and the characters of $T^{\prime}$. From $[2, \S 4]$ $B(\sigma, H)$ is a 2-block and every 2-block of $G$ is given in this form.

Fix $H$ and $\sigma$ and assume $B(\sigma, H)$ is real. If $\chi$ is the character afforded by $T^{\prime G} \in B(\sigma, H)$, then its complex conjugate $\chi^{c} \in B(\sigma, H)$. Thus $\bar{T}_{K(\Lambda)}^{\prime}$ and ${\overline{\left(T^{\prime c}\right)}}_{K(\Lambda)}$ are $G$-conjugate to $\sigma$. Since $T^{\prime c}(k)=T^{\prime}\left(k^{-1}\right)$ for all $k \in K(L) \supseteq K(\Lambda)$, it follows

Received by the editors January 15, 1983 and, in revised form, July 14, 1983.

1980 Mathematics Subject Classification. Primary 20C15, 20C20.

Key words and phrases. Characters, real characters, $p$-blocks, real $p$-blocks, 2-rational characters, modular and ordinary representations. 
that $\sigma^{\prime}$, defined by $\sigma^{\prime}(f)=\sigma\left(f^{-1}\right)$ for all $f \in K(\Lambda)$, is $G$-conjugate to $\sigma$. Since $\sigma^{\prime}(f)=\sigma\left(f^{-1}\right)$ means ker $\sigma^{\prime}=\operatorname{ker} \sigma$, there exists $y \in N(S), y$ unique modulo the inertia group $I(\sigma)$, such that $\sigma\left(y^{-1} f y\right)=\sigma\left(f^{-1}\right)$ for all $f \in K(\Lambda)$, or $y$ inverts every element of $K(\Lambda) / S$. Note that $K(\Lambda) / S$ is of odd order.

We claim that $K(H) / S$ splits over $K(\Lambda) / S$. Let $r$ be a prime and $R / S$ be the $r$-Sylow subgroup of $K(H) / S, K(H) \supseteq K(\Lambda)$, and $R_{0} / S=R / S \cap K(\Lambda) / S$. If $r=2$ then $R_{0} / S=1$. Thus let $r$ be odd, $R_{0} / S=\langle c S\rangle$ where $c S$ is of order $r^{s}$, and $R / R_{0}=\left\langle z_{1} R_{0}, \ldots, z_{n} R_{0}\right\rangle, z_{i} R_{0}$ being a basis for the abelian group $R / R_{0}$. Let $z=z_{i}$, and assume $z R_{0}$ is of order $r^{u}$ with $z^{r^{u}} \equiv c^{r^{i}}(\bmod S)$. If $u \leqslant v$, then, letting $w=z^{-1} c^{r^{--u}}$, we get $w^{r^{u}} \equiv 1(\bmod S)$. Assume $v<s$ and $u>v$. Since $[R, y] \subseteq G^{\prime}$ $\cap R \subseteq K(\Lambda) \cap R=R_{0}$, it follows that $y^{-1} z y \equiv z c^{\lambda}(\bmod S)$. Thus

$$
y^{-1} z^{r^{u}} y \equiv z^{r^{u}} c^{\lambda r^{u}} \equiv c^{-r^{r}} \quad(\bmod S),
$$

implying $\lambda r^{u} \equiv-2 r^{v}\left(\bmod r^{s}\right)$, a contradiction. Thus $K(H) / S$ splits over $K(\Lambda) / S$.

The above also implies that $c S, z_{1} S, \ldots, z_{n} S$, with the $z_{i}$ 's chosen such that $z_{i} S$ is of order $r^{u_{i}}$, form a basis for $R / S$ with $R_{0} / S=\langle c S\rangle$. (Note that if $r=2$ then $R_{0} / S=1$, and thus the above is true.) Let $y^{-1} z_{i} y \equiv z_{i} c^{\lambda},(\bmod S)$ and $\mu_{i}$ be a solution of $\lambda_{i}+2 \mu_{i} \equiv 0\left(\bmod r^{s}\right)$. If $R_{0} / S=1$ (as in the case of $\left.r=2\right)$ let $\lambda_{i}=\mu_{i}=0$. For each $r$-Sylow subgroup $R / S$ of $K(H) / S$, we fix such a set of $z_{i}$ 's.

Our aim now is to construct the character $\theta$ of the theorem. Let $T$ be a (complex) linear representation of $K(\Lambda), \bar{T}=\sigma, \operatorname{ker} T \cap G^{\prime}=H$, and $T(k)=1$ for every 2-element $k$ of $K(\Lambda)$. Then $\operatorname{ker} T=\operatorname{ker} \sigma=S$. Extend $T$ as $T^{\prime}$ to all elements of $R K(\Lambda)$ for every $r$-Sylow subgroup $R K(\Lambda) / K(\Lambda)$ of $K(H) / K(\Lambda)$ and then to all elements of $K(H)$ as follows: If $R_{0} / S=1$ (as in the case of $r=2$ ) then let $T^{\prime}\left(z_{i}\right)=1$ for all $i$. If $R_{0} / S=\langle c S\rangle \neq 1$ (notations as above) let $T^{\prime}\left(z_{i}\right)=T(c)^{\mu_{i}}$. Now if $k \in K(H)$ then $k=a\left(\Pi^{\prime}\left(\Pi_{i} z_{i}^{e_{i}}\right)\right)$, $\Pi^{\prime}$ running over all primes $r$ dividing $|K(H) / S|$ and $a \in K(\Lambda)$. Define $T^{\prime}(k)=T(a)\left(\Pi^{\prime}\left(\Pi_{i} T^{\prime}\left(z_{i}\right)^{e_{i}}\right)\right)$. It is easy to show that $T^{\prime}$ is a linear representation of $K(H)$. Now $T^{\prime} \in R(K(\Lambda), S, K(H))$, as in the notations of [1, p. 100], and hence $T^{\prime G}$ is irreducible. Since $\left(\bar{T}^{\prime}\right)_{K(\Lambda)}=\sigma$ we have $T^{\prime} G \in B(\sigma, H)$. Let $\theta$ be the character of $T^{\prime G}$. Then $\theta$ is 2-rational and since $\operatorname{ker} \theta$ contains the defect group of $B(\sigma, H), \theta$ is of height zero in $B(\sigma, H)$. It remains to show that $Q(B(\alpha, H))=Q(\theta)$.

Now $I\left(T^{\prime}\right)=K(H), I\left(T^{\prime}\right)$ the inertia group of $T^{\prime}$. We claim that $I(\sigma)=K(H)$. Let $x \in I(\sigma)$. Then for any prime $r, T\left(x^{-1} c x\right)=T(c)$. (Here $c, z_{i}$ depend on $r$ as in the notations above.) If $R_{0} / S=1$, then $x^{-1} z x=z(\bmod S)$ for any $z=z_{i} \in R$. Assume for some (odd) $r, R_{0} / S=\langle c S\rangle \neq 1$, and for some generator $z S$ of $R / S$, $x^{-1} z x \equiv z c^{\delta}(\bmod S)$. Since $x^{-1} c x \equiv c(\bmod S), x \neq y$. Thus we may assume $x y \equiv y x a$ $(\bmod S), a \in K(\Lambda)$. It follows that $(x y)^{-1} z(x y) \equiv(y x)^{-1} z(y x)(\bmod S)$. After a short computation we have $z c^{\lambda} c^{-\delta} \equiv z c^{\delta} c^{\lambda}(\bmod S), \lambda$ defined as above. Thus $2 \delta \equiv 0\left(\bmod r^{s}\right)$, and since $r \neq 2, \delta \equiv 0\left(\bmod r^{s}\right)$ or $x \in K(H)$. Thus $I(\sigma)=K(H)$ $=I\left(T^{\prime}\right)$.

Let $\zeta$ be a primitive $|G|$ th root of unity. Then $Q(\chi) \subseteq Q(\zeta)$ for all irreducible characters $\chi$ in $B(\sigma, H)$. Let $\tau \in \mathcal{G}(Q(\zeta) / Q)$ and assume $B(\sigma, H)^{\tau}=B(\sigma, H)$. This 
implies both $T^{\prime G}$ and $\left(T^{\prime G}\right)^{\tau}$ are in $B(\sigma, H)$ and thus $\sigma=\bar{T}=\bar{T}_{K(\Lambda)}^{\prime}$ and $\bar{T}^{\tau}=$ $\left(\bar{T}^{\prime \tau}\right)_{K(\Lambda)}$ are $G$-conjugate or $T$ and $T^{\tau}$ are $G$-conjugate. Assume $T^{\tau}=T^{g}$ for some $g \in G$. Since $\operatorname{ker} T=\operatorname{ker} T^{g}=S$, it follows that $g \in N(S)$. Now, for the prime $r$, $r \neq 2$, the group $\langle y, g\rangle$ induces a (cyclic) automorphism subgroup on $R_{0} / S=\langle c S\rangle$. That is, there is an element $h_{r} \in N(S)$ such that $\left\langle h_{r}\right\rangle$ induces the same automorphism group on $R_{0} / S$ as $\langle y, g\rangle$. If $h_{r}$ induces an automorphism of order $n$ on $R_{0} / S$ then $n$ is even and $h_{r}^{n / 2}$ inverts every element of $R_{0} / S$. Assume $h_{r}^{-1} c h_{r} \equiv c^{\alpha}(\bmod S)$. Then $r \nmid \alpha-1, g^{-1} c g \equiv c^{\alpha^{t}}(\bmod S)$ and $\alpha^{n / 2} \equiv-1\left(\bmod r^{s}\right)$. Assume $h_{r}^{-1} z h_{r} \equiv z c^{\nu}$ $(\bmod S), g^{-1} z g \equiv z c^{\delta}(\bmod S)$, where $z S$ is a generator of $R / S$ as defined above. Since

$$
\begin{aligned}
\left(y h_{r}^{n / 2}\right)^{-1} z\left(y h_{r}^{n / 2}\right) & \equiv\left(h_{r}^{n / 2} y\right)^{-1} z\left(h_{r}^{n / 2} z\right) \quad(\bmod S) \\
\left(g h_{r}^{t}\right)^{-1} z\left(g h_{r}^{t}\right) & \equiv\left(h_{r}^{t} g\right)^{-1} z\left(h_{r}^{t} g\right) \quad(\bmod S)
\end{aligned}
$$

and

$$
h_{r}^{-e} z h_{r}^{e} \equiv z c^{\nu\left(\alpha^{e-1}+\cdots+1\right)}(\bmod S) \quad \text { for any } e
$$

it follows that

$$
\lambda \equiv \nu\left(\alpha^{n / 2-1}+\cdots+1\right)\left(\bmod r^{s}\right) \text { and } \delta \equiv \nu\left(\alpha^{t-1}+\cdots+1\right)\left(\bmod r^{s}\right) .
$$

That is, $\langle y, g\rangle$ induces a cyclic automorphism group on $R / S$.

Recalling that $T^{\prime}(z)=T(c)^{\mu}, \lambda+2 \mu \equiv 0\left(\bmod r^{s}\right)$, and $T^{\tau}=T^{g}$, we have

$$
T^{\prime}(z)^{\tau}=\left[T(c)^{\mu}\right]^{\tau}=T\left(g^{-1} c g\right)^{\mu}=T(c)^{\mu \alpha^{\prime}} .
$$

Also

$$
T^{\prime}\left(g^{-1} z g\right)=T^{\prime}\left(z c^{\delta}\right)=T^{\prime}(z) T(c)^{\delta}=T(c)^{\mu+\delta} .
$$

We need to prove $\mu \alpha^{t} \equiv \mu+\delta\left(\bmod r^{s}\right)$ or $\mu\left(\alpha^{t}-1\right) \equiv \delta\left(\bmod r^{s}\right)$. Since $\alpha^{n / 2} \equiv-1$ $\left(\bmod r^{s}\right)$, we have

$$
2 \nu\left(\alpha^{t}-1\right) \equiv-\nu\left(\alpha^{t}-1\right)\left(\alpha^{n / 2}-1\right) \quad\left(\bmod r^{s}\right) .
$$

Since $r \nmid \alpha-1$, we have

$$
2 \nu\left(\alpha^{t-1}+\cdots+1\right) \equiv-\nu\left(\alpha^{t}-1\right)\left(\alpha^{n / 2}+\cdots+1\right)\left(\bmod r^{s}\right) .
$$

Thus

$$
2 \delta \equiv-\lambda\left(\alpha^{t}-1\right)\left(\bmod r^{s}\right) \quad \text { or } \quad \delta \equiv \mu\left(\alpha^{t}-1\right)\left(\bmod r^{s}\right)
$$

as desired. Thus $T^{\prime \tau}=T^{\prime g}$ or $T^{\prime \tau}$ and $T^{\prime}$ are $G$-conjugate. This implies $T^{\prime G}$ and $\left(T^{\prime \tau}\right)^{G}$ are equivalent or $\theta^{\tau}=\theta$. The proof is complete.

The above implies

COROllaRY. Let $B$ be a 2-block of a finite metabelian group and assume $B$ contains all the $Q$-conjugates of some irreducible character. Then $B$ contains an irreducible rational character of height zero. 


\section{REFERENCES}

1. B. G. Basmaji, Monomial representations and metablien groups, Nagoya Math. J. 35 (1969), 99-107. MR 39 \# 5709.

2. Modular representations of metabelian groups, Trans. Amer. Math. Soc. 169 (1972), 389-399. MR 46 \#9153.

3. On the field of a 2-block, Proc. Amer. Math. Soc. 83 (1981), 471-475.

4. R. Gow, Real-valued and 2-rational group characters, J. Algebra 61 (1979), 388-413.

Department of Mathematics, California State University, los Angeles, California 90032 